\title{
RAPID CREATION OF A TEMPORARY ISOLATION WARD for Patients With Severe Acute Respiratory SYNDROME IN TAIWAN
}

\author{
Chang-Phone Fung, MD, MSc; Tsorng-Liu Hsieh, MD; Kuang-Huan Tan, MD; Chin-Hui Loh, MD, PhD; Jiunn-Sheng Wu, MD; \\ Chien-Chung Li, MD; Feng-Yee Chang, MD, PhD; L. K. Siu, PhD; Moh-Yun Yen, MD; Lih-Shinn Wang, MD, MSc; \\ Wing-Wai Wong, MD; Wei-Fong Kao, MD; Jia-Hui Hsu, BS; Ting-Hsiang Lin, PhD; Fu-Yuan Huang, MD; Chen-Hsen Lee, MD
}

\begin{abstract}
OBJECTIVES: To rapidly establish a temporary isolation ward to handle an unexpected sudden outbreak of severe acute respiratory syndrome (SARS) and to evaluate the implementation of exposure control measures by healthcare workers (HCWs) for SARS patients.

DESIGN: Rapid creation of 60 relatively negative pressure isolation rooms for 196 suspected SARS patients transferred from 19 hospitals and daily temperature recordings of 180 volunteer HCWs from 6 medical centers.

SETTING: A military hospital.

RESULTS: Of the 196 patients, 34 (17.3\%) met the World Health Organization criteria for probable SARS with positive results of serologic testing for SARS-associated coronavirus (SARS-CoV), reverse transcriptase polymerase chain reaction (RT-PCR) from nasopharyngeal or throat swabs for SARS-CoV, or both. Seventy-four patients had suspected SARS based on unpro-

tected exposure to SARS patients; three of them had positive results on RT-PCR but negative serologic results. The remaining 88 patients did not meet the criteria for a probable or suspected SARS diagnosis. Of the 34 patients with probable SARS, 13 were transferred to medical centers to receive mechanical ventilation due to rapid deterioration of chest $\mathrm{x}$-ray results, and three patients died of SARS despite intensive therapy in medical centers. During the study period, one nurse developed probable SARS due to violation of infection control measures, but there was no evidence of cross-transmission to other HCWs.

CONCLUSIONS: Despite the use of full personal protection equipment, the facility failed to totally prevent exposures of HCWs to SARS but minimized the risk of nosocomial transmission. Better training and improvements in infection control infrastructure may limit the impact of SARS (Infect Control Hosp Epidemiol 2004;25:1026-1032).
\end{abstract}

Severe acute respiratory syndrome (SARS) is caused by a novel highly contagious coronavirus, which spread from Guandong, China, to Hong Kong, Singapore, Vietnam, Canada, and Taiwan within a short period. ${ }^{1-6}$ Through July 4,2003 , a total of 8,439 probable SARS cases were reported from 32 countries with a case-fatality rate of $9.6 \%$.

The first probable SARS case in Taiwan was identified on March 13, 2003. The patient, a 54-year-old Taiwan native, had traveled to Guandong Province in China in early February and returned to Taipei via Hong Kong on February 25 with fever, myalgia, and a dry cough. ${ }^{6}$ His wife and son also developed the same symptoms a few days later. All three affected family members were isolated in negative-pressure rooms in a medical center with full infection precautions. From March 14 to April 21, Taiwan reported 28 probable cases of SARS, which were still successfully monitored and controlled. ${ }^{8}$

Between April 22 and 29, 2003, two major nosocomial SARS outbreaks occurred. One was at Taipei Municipal Hoping Hospital and the other was at Jen-Chi Hospital. As a result of these outbreaks, 137 patients (including 45 healthcare workers [HCWs]) met the case definition for probable SARS issued by the World Health Organization (WHO) on April 20, 2003, ${ }^{9}$ resulting in more than 10,000 individuals being placed in home quarantine. Because of a shortage of isolation rooms for use in these outbreaks and the impossibility of constructing a large number of negative-pressure rooms within a short time, the government quickly designated Sung-Shan Military Hospital as a temporary isolation hospital for all suspected SARS patients. We report our experience in the rapid creation and use of a temporary isolation ward in this hospital prior to the construction of standard isolation wards.

Drs. Fung and Wong are from the Section of Infectious Diseases and Drs. Lee and Kao are from the Department of Emergency Service, Taipei Veterans General Hospital and National Yang-Ming University, Taipei, Taiwan. Drs. Hsieh, Wu, and Li are from the Department of Internal Medicine and Ms. Hsu and Dr. Tan are from the Committee of Infection Control, Sung-Shan Military Hospital, Taipei, Taiwan. Drs. Chang and Lo are from the Division of Infectious Diseases, Tri-Service General Hospital, Taipei, Taiwan. Dr. Siu is from the Division of Clinical Research, National Health Research Institute, Taipei, Taiwan. Dr. Yen is from the Department of Emergency Service, Kaohsiung Veterans General Hospital, Kaohsiung, Taiwan. Dr. Wang is from the Division of Infectious Diseases, Buddhist Tzu Chi General Hospital, Hwalien, Taiwan. Dr. Lin is from the Division of Laboratory Research and Development, Center for Disease Control, Department of Health, Taipei, Taiwan. Dr. Huang is from the MacKay Memorial Hospital, Taipei, Taiwan.

Address reprint requests to Chen-Hsen Lee, MD, Taipei Veterans General Hospital, 201, Section 2, Shih-Pai Road, Taipei, 11217, Taiwan.

The authors thank all of the healthcare workers and volunteers from Taipei Veterans General Hospital, Chang Gung Memorial HospitalLinKou Medical Center, MacKay Memorial Hospital, Tri-Service General Hospital, Cathy General Hospital, Shin Kong Wu Ho-Su Memorial Hospital, and Buddhist Tzu Chi General Hospital for their dedication in taking care of patients with SARS. 


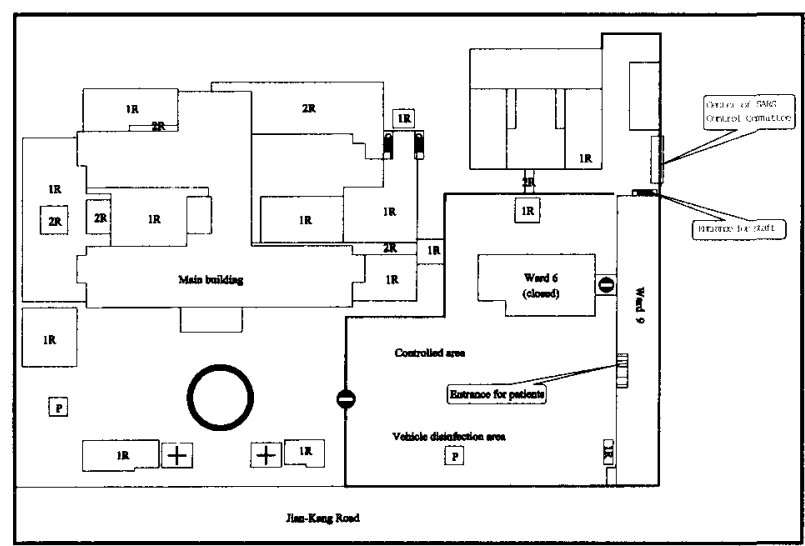

FIGURE 1. The location of ward 9 in Sung-Shan Military Hospital. SARS = severe acute respiratory syndrome.

\section{METHODS \\ Hospital Location}

Sung-Shan Military Hospital, located in the eastern area of Taipei, is a 450-bed facility that provides medical services to soldiers and civilians. The hospital has several separate wards (satellite buildings) around the main building. Of these, the ward 9 building is farthest from other buildings and from public areas. Constructed 30 years ago, ward 9 contains four stories with independent ventilation for each floor. There are two separate entrances into the first floor and another entrance on the second floor connecting it with ward 6 (Fig. 1). Each floor has 20 private rooms with independent handwashing and toilet facilities except the first floor, which was designed as a physical rehabilitation area.

\section{Establishment of the Temporary Isolation Ward}

To begin, we moved all patients and unnecessary equipment to other buildings and installed a commercialgrade (three-wing panel fan, $14 \mathrm{in}, 65 \mathrm{~W}$, and $60 \mathrm{~Hz}$ ) exhaust fan above the window in each private room on the second, third, and fourth floors (Fig. 2). We then girded the doors of the private rooms with a heavy cloth to plug the gap around the doors so as to block the air from the corridor and create a relatively negative pressure inside the private rooms from 0.028 to 0.07 in water gauge compared with the nurse station and corridor ( 0 in water gauge) based on magnehelic gauge readings. The stairways between each floor were closed to prevent cross-ventilation. Each floor had independent ventilation equipment, the ducts of which had been installed in the crawlspace of a drop ceiling in 1974 .

The first floor was divided into a clean zone for handwashing, changing into and out of private clothes, donning double layers of personal protective equipment (PPE), and recording name and entry and exit times; an intermediate zone for removing the inner layer of PPE, handwashing, and showering; and a contaminated zone for entering the isolation rooms and removing the outer layer of PPE when the HCWs were returning to the

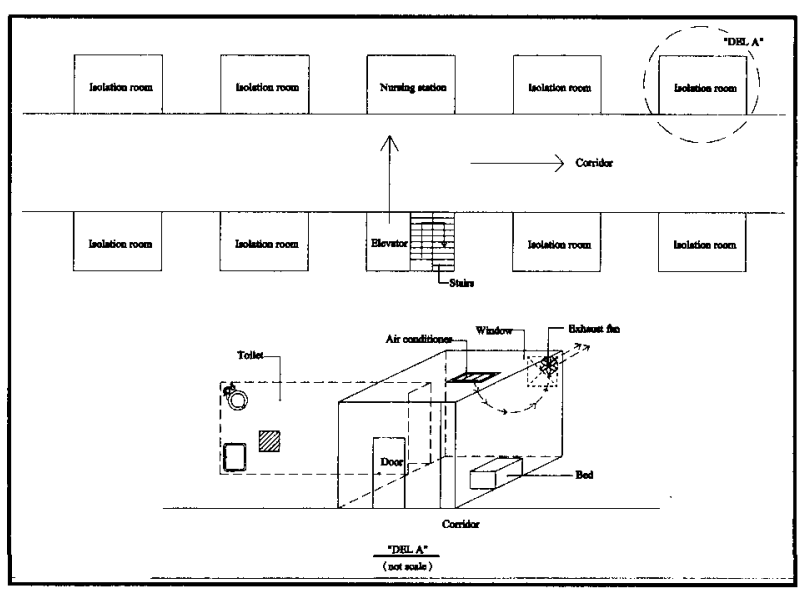

FIGURE 2. Floor plan of ward 9 after conversion from an open unit to isolation rooms. $\mathrm{DEL}=$ detail.

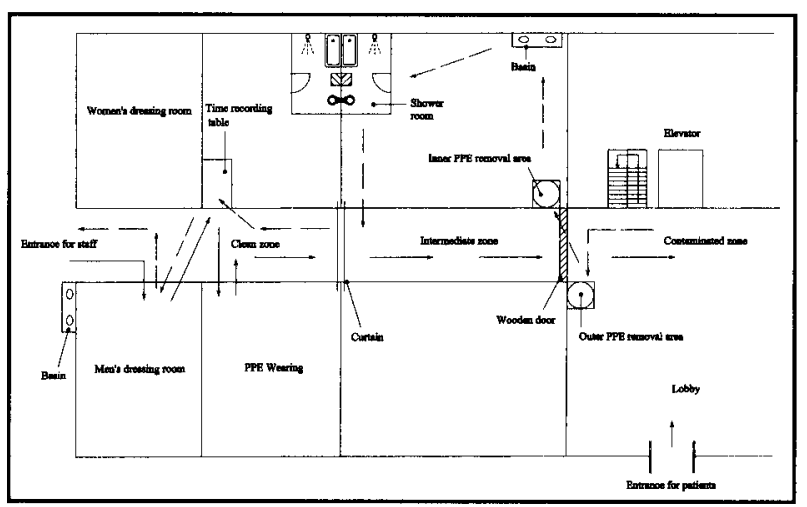

FIGURE 3. Work flow on the first floor of ward 9 . The solid line indicates the direction of entering the isolation ward and the dotted line indicates the direction of leaving the isolation ward. PPE = personal protective equipment.

intermediate zone (Fig. 3). A movable curtain along the corridor was set up to separate the clean zone and the intermediate zone, and a movable wooden door was constructed to separate the intermediate zone and the contaminated zone. The environment was disinfected with sodium hypochlorite $(1,000 \mathrm{ppm})$ twice a day, and $75 \%$ alcohol was used for the metallic surfaces. The entire ward was completed and ready for use within 2 days.

\section{Sources of Medical Staff}

The medical staff included infectious diseases specialists, chest physicians, experienced residents and nurses, and radiology technicians. Most members of the medical staff were selected from six medical centers in Taipei along with a few volunteers from other hospitals. All HCWs including administrators and ward cleaners received 3 hours of intensive training in infection control measures before they were regarded as qualified to enter the isolation ward. They also received a training course in standard procedures for wearing and removing PPE such 
as an N-95 respirator, face shield or goggles, cap, gown, and two pairs of gloves and shoe covers. A huge poster of standard procedures and a large mirror were placed on the wall to aid staff in following procedures. Staff were also asked to double-check each other for compliance with these procedures before entering the isolation ward. The temperature of each staff member was recorded daily before entering the isolation ward.

\section{Source of Patients and Case Definitions}

Patients with fever (temperature $>38^{\circ} \mathrm{C}$ ) were initially evaluated by the emergency department practitioners of medical centers and regional hospitals who then faxed patients' clinical data including chest x-ray findings and complete blood cell count to Sung-Shan Military Hospital for further evaluation. After approval by the Sung-Shan Military Hospital coordination center, patients were prepared for transfer. Patients with severe dyspnea were transferred to medical centers directly. SARS was defined according to the definitions issued by the WHO on May 1 , 2003. ${ }^{9}$ A suspected case was an individual presenting with fever (temperature $>38^{\circ} \mathrm{C}$ ), cough, or breathing difficulty who had a history of travel to an area associated with SARS transmission or close contact (including having cared for, lived with, or had direct contact with respiratory secretions or body fluids) with an individual who was a suspected or probable case of SARS ${ }^{9,10}$ A probable case was a suspected case with evidence of infiltrates consistent with pneumonia or respiratory distress syndrome on chest $\mathrm{x}$-ray, a positive result for SARS-associated coronavirus (SARS-CoV) from nasopharyngeal or throat swab by reverse transcriptase polymerase chain reaction (RT-PCR), or a positive result of serologic testing by means of both enzyme-linked immunosorbent assay and immunofluorescent assay to detect SARS-CoV antibodies from the saved sera by the Division of Laboratory Research and Development, Center for Disease Control of Taiwan. The exclusion criteria were an alternative diagnosis that can fully explain the illness and a negative result on RT-PCR or serologic study.

\section{Patient Transport}

All suspected SARS patients were transported by ambulance after reporting to the Center for Disease Control of Taiwan and the local public health authority. Each vehicle allowed only one patient per transfer. Before entering the vehicle, the patient was asked to wear disposable underwear, an N-95 mask without an exhaust valve, a cap, a gown, gloves, and shoe covers. The driver and medical staff were asked to wear N-95 respirators plus surgical masks outside and double layers of PPE. The window between the driver and the patient compartment was closed tightly before the patient was brought inside the vehicle. The outside layers of gloves of the driver and medical staff were washed immediately with $75 \%$ alcohol before and after patient contact or touching contaminated items and washed with $20 \%$ chlorhexidine after removal. During transportation, all windows in the patient compartment were closed. No patient required oxygen during transport. Patients who required oxygen support would have been immediately transported to medical centers with an oxygen mask prepared in the ambulance. Patients were guided to the assigned ward by fully protected medical staff. After patient transportation was completed, the entire transport vehicle was cleaned and disinfected immediately by a cleaning worker wearing PPE before it was returned to service.

\section{Management of Suspected SARS Cases in the Ward}

The entire ward was regarded as a contaminated area. Visitors were not allowed to enter the ward. Patients were also not allowed to leave their rooms, and the doors were closed all day. Patients were notified to don a mask before staff entered their rooms. All HCWs changed their outside layer of PPE after finishing their medical tasks for each patient. All patients underwent complete blood cell counts and chest $x$-rays daily. Patients with probable or suspected SARS received oral ribavirin $(600 \mathrm{mg}$ twice daily) and levofloxacin (500 $\mathrm{mg}$ daily) for 7 days if their chest x-ray yielded abnormal findings. Methylprednisolone was prescribed only for those with high fever (body temperature > $39^{\circ} \mathrm{C}$ ). Patients were transferred to one of the large medical centers with negative-pressure facilities for intensive care or mechanical ventilation if their chest x-rays showed rapid deterioration or if they had severe respiratory failure (arterial partial pressure of oxygen $\left[\mathrm{PaO}_{2}\right]<50 \mathrm{~mm} \mathrm{Hg}$ ).

A portable radiology machine was placed in the ward for taking daily chest x-rays. The chest film boxes were carefully wrapped with double plastic bags before sending them out to be assessed by radiologists, and the inside layer of the plastic bag was disinfected with $75 \%$ alcohol. All necessary materials were sent to the patients' entrance and then taken by ward staff into the ward. All waste materials were placed in double red biohazard bags and all liquid specimens were securely sealed and placed upright in a transport box. Patients urinated and defecated into bedpans in which the waste was treated with $5,000 \mathrm{ppm}$ of sodium hypochlorite (1:10 dilution of bleach solution) for 30 minutes and then carefully disposed of into the sanitary sewer. The bedpan was then rinsed with tap water and soaked in 1,000 ppm (1:50 dilution) of bleach solution for 15 minutes before the contents were disposed of into the sanitary sewer.

The environment was disinfected daily with sodium hypochlorite $(1,000 \mathrm{ppm})$ except for metallic surfaces, which were disinfected with $75 \%$ alcohol. After a patient was discharged, the room was disinfected with ultraviolet light for 2 hours, the floor was cleaned with sodium hypochlorite $(1,000 \mathrm{ppm})$, and $75 \%$ alcohol was used for cleaning the metallic surfaces.

\section{RESULTS}

From April 27 to May 22, 2003, a total of 196 patients with suspected SARS were admitted for treatment. Their median age was 37 years (range, 3 to 87 
TABLE 1

Number of Patifnts With Severe Acute Respiratory Syndrome Transferrei From 19 Hospitals and Their Diagnostic Status

\begin{tabular}{|c|c|c|c|c|c|c|}
\hline \multirow[b]{3}{*}{ Hospital } & \multicolumn{6}{|c|}{ No. of Patlents } \\
\hline & \multirow[b]{2}{*}{ Transferred } & \multirow[b]{2}{*}{ Probable } & \multirow[b]{2}{*}{ Suspected } & \multirow[b]{2}{*}{ Excluded } & \multicolumn{2}{|c|}{ Positive Results for } \\
\hline & & & & & RT-PCR & Serology* \\
\hline A & 43 & 0 & 16 & 27 & 1 & 0 \\
\hline B & 36 & 16 & 15 & 5 & 12 & 16 \\
\hline $\mathrm{C}$ & 34 & 5 & 10 & 19 & 5 & 5 \\
\hline $\mathrm{D}$ & 31 & 6 & 14 & 11 & 5 & 6 \\
\hline $\mathrm{E}$ & 10 & 2 & 8 & 0 & 2 & 2 \\
\hline $\mathrm{F}$ & 9 & 0 & 3 & 6 & 1 & 0 \\
\hline $\mathrm{G}$ & 5 & 0 & 2 & 3 & 1 & 0 \\
\hline $\mathrm{H}$ & 5 & 1 & 2 & 2 & 1 & 1 \\
\hline I & 5 & 1 & 0 & 4 & 1 & 1 \\
\hline $\mathrm{J}$ & 4 & 1 & 0 & 3 & 0 & 1 \\
\hline $\mathrm{K}$ & 4 & 1 & 2 & 1 & 1 & 1 \\
\hline $\mathrm{L}$ & 3 & 0 & 0 & 3 & 0 & 0 \\
\hline M & 1 & 1 & 0 & 0 & 0 & 1 \\
\hline $\mathrm{N}$ & 1 & 0 & 0 & 1 & 0 & 0 \\
\hline 0 & 1 & 0 & 0 & 1 & 0 & 0 \\
\hline$P$ & 1 & 0 & 0 & 1 & 0 & 0 \\
\hline$Q$ & 1 & 0 & 1 & 0 & 0 & 0 \\
\hline $\mathrm{R}$ & 1 & 0 & 1 & 0 & 0 & 0 \\
\hline S & 1 & 0 & 0 & 1 & 0 & 0 \\
\hline Total & 196 & 34 & 74 & 88 & 30 & 34 \\
\hline
\end{tabular}

RT-PCR = reverse transcriptase polymerase chain reaction.

*Serologic studies included enzyme-linked immunosorbent assay and immunofluorescent assay.

years). The male-to-female ratio was 0.85 (91 per 106). Of the 196 patients, $46(23.5 \%)$ were transferred from the two hospitals in Taipei that had nosocomial SARS outbreaks, whereas the remaining patients $(76.5 \%$; 150 of 196) were transferred from emergency departments of other hospitals (Table 1). Specimens from nasopharyngeal or throat swab for RT-PCR were carefully tested for all suspected SARS patients, and 30 samples had positive results for SARS-CoV. Serologic studies revealed a total of 34 patients had positive results by enzyme-linked immunosorbent assay, immunofluorescent assay, or both.

Table 2 presents the WHO classification of SARS patients by HCW or non-HCW status and by contact history. Of the 196 patients, 34 (17.3\%) had probable SARS according to the WHO criteria and positive results of serologic testing confirmed by the Center for Disease Control of Taiwan. Of these 34 probable cases, 27 (79.4\%) also had positive RT.PCR results. These patients were reported to the WHO representative immediately when their initial RT-PCR results were obtained. Seventy-four patients had suspected SARS because of unprotected exposure to SARS patients. Although three of them had positive RT-PCR results, their serologic tests showed negative results on enzyme-linked immunosorbent assay or immunofluorescent assay study of the saved sera. SARS was ruled out in the remaining 88 patients because their specimens showed negative results on RT-PCR or serologic study. Of these patients, $39.8 \%$ (35 of 88 ) had no contact history with SARS patients, had no active lung lesion on chest $\mathrm{x}$-rays, and had progressive subsidence of fever after symptomatic treatment of their condition as a common cold. Although 38 patients $(43.2 \%$; 38 of 88 ) had pneumonic patches or interstitial infiltrations on chest $x$-rays, they had good responses to antibiotic therapy (including roxithromycin, levofloxacin, ceftriaxone, and imipenem) and were soon shown to have pneumococcal pneumonia, Mycoplasma pneumonia, or legionnaires' disease. The remaining 15 patients $(17.1 \% ; 15$ of 88$)$ without findings on chest x-ray were finally shown to have acute tonsillitis, acute bronchitis, or infectious diarrhea.

Of the 196 patients, $51(26 \%)$ were hospital HCWs including 7 physicians, 26 nurses, 12 medical technicians, and 6 laundry workers. Most of them (72.5\%; 37 of 51) were transferred from the two hospitals with SARS outbreaks in Taipei. There were $17 \mathrm{HCWs}(50 \%$; 17 of 34$)$ who had documented cases of probable SARS.

Table 3 lists the outcomes of all suspected SARS patients who stayed in the isolation ward. The mean duration of stay in the isolation ward for those with probable SARS who required transfer to other medical centers was 
TABLE 2

Classification of Patients Suspected to Have Severe Acute Respiratory Syndrome as Healthcare Workers or NON-HEALTHCARE WORKERS

\begin{tabular}{|c|c|c|c|c|c|}
\hline & \multicolumn{5}{|c|}{ No. of Patients $(\%)$} \\
\hline & & & & \multicolumn{2}{|c|}{ Non-Healthcare Worker } \\
\hline & \multicolumn{3}{|c|}{ Healthcare Worker } & With Contact & Whthout Contact \\
\hline & Physiclan & Nurse & Other & History & Hlstory \\
\hline Probable SARS ( $(\mathbf{n}=34)$ & $1(2.94)$ & $10(29.4)$ & $6(17.7)$ & $17(50)$ & 0 \\
\hline Suspected SARS $(n=74)$ & $6(8.1)$ & $16(21.6)$ & $9(12.2)$ & $43(58.1)$ & 0 \\
\hline Excluded $(n=88)$ & 0 & 0 & $3(3.4)$ & $50(56.8)$ & $35(39.8)$ \\
\hline Total $(n=196)$ & $7(3.6)$ & $26(13.3)$ & $18(9.2)$ & $110(56.1)$ & $35(17.8)$ \\
\hline
\end{tabular}

SARS - severe acute respiratory syndrome.

TABLE 3

Clinical Outcome of Pattents During Their Stay in the Temporary Isolation Ward

\begin{tabular}{|c|c|c|c|c|c|}
\hline & \multirow{2}{*}{$\begin{array}{l}\text { Mean No. of } \\
\text { Hospltal-Days } \\
\text { (Range) for Those } \\
\text { Not Transferred }\end{array}$} & \multirow{2}{*}{$\begin{array}{l}\text { Transferred to } \\
\text { Medical Center }\end{array}$} & \multirow{2}{*}{$\begin{array}{c}\text { Dlscharged } \\
\text { for Home } \\
\text { Quarantine }\end{array}$} & \multicolumn{2}{|c|}{ Outcome } \\
\hline & & & & Recovered & Dled \\
\hline Probable SARS $(n=34)$ & $9.4(6-24)$ & $13^{\star}$ & 21 & 31 & 3 \\
\hline Suspected SARS $(n=74)$ & $8.5(5-28)$ & 6 & 68 & 74 & 0 \\
\hline Excluded $(\mathbf{n}=88)$ & $7.2(3-16)$ & 1 & 87 & 88 & 0 \\
\hline
\end{tabular}

short (3.6 days; range, 1 to 5 days). However, the mean number of hospital-days for those not transferred (probable group, 9.4 days) was greater than that for those for whom SARS was excluded (7.2 days). Of the 34 probable SARS patients, 13 were transferred to a medical center for mechanical ventilation due to rapid deterioration of chest $x$-ray results, and three died of SARS despite intensive therapy in medical centers.

During the study period, a total of $180 \mathrm{HCWs}$ entered the isolation ward, including 16 physicians, 144 nurses, 3 administrators, 6 radiology technicians, and 11 ward cleaners. The mean number of working days for staff working in the isolation ward was 5.1 (range, 1 to 16 days). Three nurses had temperatures greater than $38^{\circ} \mathrm{C}$ during different periods. They were isolated immediately, and 54 of their colleagues who worked with them at the same time were also simultaneously quarantined for 10 days in the hospital or at home. Of the three nurses who developed fever, SARS was excluded quickly in one patient due to symptoms and signs of urinary tract infection without any lung lesion; another had suspected SARS based on the presentation of fever and leukopenia (white blood cell count once decreased to $2,000 / \mathrm{mm}^{3}$ ), but fever subsided after antibiotic treatment $(100 \mathrm{mg}$ of oral roxithromycin every 12 hours) and both RT-PCR and serologic testing yielded negative results for SARS$\mathrm{CoV}$. The third nurse was classified as having probable
SARS based on the positive results of RT-PCR and serologic testing. Tracing her history, this nurse recalled that a few days prior to the onset of fever, she had once carelessly touched her eyes with her hands while taking off her PPE before she had washed her hands. Fortunately, there was no evidence that she had spread the virus to other individuals and all of her colleagues who had worked with her at the same time were healthy during their quarantine periods.

\section{DISCUSSION}

In the management of SARS patients, isolation inside a negative-pressure room is generally accepted as a measure for protecting HCWs from nosocomial infection. During the period from April 22 to May 28, 2003, a large SARS outbreak occurred that involved many suspected SARS cases at a time when all standard negativepressure rooms in the entire city were already full of SARS patients. Urgent planning for the prevention of further transmission became necessary. Redistribution of suspected SARS patients into different hospitals would have substantially increased the burden and the risk of infection to HCWs.

In Hong Kong and Taiwan, the initial redistribution of SARS patients into different hospitals resulted in a high risk of nosocomial infection, and many $\mathrm{HCW}$ sere infected due to unprotected exposure to suspected SARS 
patients. By contrast, Singapore successfully controlled the number of probable SARS cases by sending suspected cases to a designated hospital, resulting in a lack of nosocomial transmission during the outbreak period. ${ }^{4}$ It considered the conversion of a general ward to a temporary isolation ward to be an important step in controlling a sudden large outbreak of SARS. A study from Beijing also demonstrated that a designated SARS hospital had the advantage of ensuring proper infection control practices, unidirectional airflow rooms, and proper patient triage and flow. ${ }^{11}$ In addition, previous studies suggested that shortening the time from clinical onset to admission reduces the risk of transmission..$^{12,13}$ Thus, the rapid creation of a designated SARS hospital helps reduce the risk of nosocomial SARS transmission.

Sung-Shan Military Hospital has now been converted into a SARS hospital and is equipped with 102 standard negative-pressure rooms for SARS patients. Sung-Shan Military Hospital showed great efficiency in reorganizing itself within 14 days, and it now serves as the first hospital specifically designated for the treatment of SARS patients as well as other severe epidemic outbreaks. However, the need for a single SARS hospital remains somewhat controversial because it could limit transmission in the designated SARS hospital but still result in outbreaks in other hospitals lulled into thinking that they were free of SARS. Fortunately, this did not occur in Taipei. A few Taipei hospitals were designated to care for SARS, and a series of extensive infection control courses were required for all $\mathrm{HCWs}$ in the regional hospitals and medical centers in Taiwan.

Although the WHO recommended that the hospital ventilation system should be turned off and that windows should be opened if an independent air supply was unavailable, ${ }^{14}$ this was not done for the following reasons: ward 9 of the Sung-Shan Military Hospital had a noncentralized ventilation system, and the weather in May was so hot and humid that febrile patients would suffer without air conditioning in such conditions.

Initially, Taiwan enjoyed a brief period of success in the treatment of the first imported case of SARS in a teaching hospital and mistakenly interpreted the situation as evidence of effective containment and excellent clinical care ${ }^{6}$ The health authorities also had a false sense of safety and became lax in their use of containment measures. ${ }^{15}$ Because there was a lack of alertness and coordination of the health authorities as well as a shortage of isolation wards and sufficient PPE for the medical staff, the nosocomial spread of SARS was repeated in several major hospitals in northern cities in Taiwan and then spread to the south in a short period, resulting in $20 \%$ of SARS involving HCWs after care of SARS patients. ${ }^{16}$ As of May 29, 2003, Taiwan had the world's third largest outbreak of SARS infection. These findings indicate that preexisting knowledge and training regarding infection control was limited and likely played a key role in the spread of this disease. Improvements in infection control infrastructure and an emphasis on training regarding infection control mea- sures were crucial for controlling SARS and could help with future outbreaks. After learning many painful lessons regarding the shortage of PPE, the government health authority now ensures the supply, allocation, and delivery of medical masks and protective suits for medical staff on the frontline.

In our isolation ward, one nurse developed probable SARS due to a break in the technique of recommended infection control practices, making the incidence of nosocomial SARS infection $0.6 \%$ ( 1 of 180 ) for medical staff with access to adequate PPE. There was no evidence of a nosocomial SARS outbreak or cross-transmission among $\mathrm{HCWs}$ in the Sung-Shan Military Hospital during the study period.

In countries with reported cases of SARS, HCWs were at high risk of SARS infection. General protection such as the use of surgical masks, gowns, and gloves and handwashing for emergency department and outpatient units is becoming a necessity in our daily practice. ${ }^{17}$ In retrospect, the value of several control measures undertaken by the SARS Control Committee in the Sung-Shan Military Hospital remains inconclusive, such as having all suspected SARS patients wear an N-95 mask and PPE during transport. However, the requirement for overprotection was made to possibly further control the risk of transmission, especially when facing an unknown disease with an unexpectedly large outbreak. Although we failed to prevent accidental exposure of HCWs to SARS, the possibility of nosocomial transmission within the designated facility was minimized. This information may be useful for infection control personnel or government health officials as they face potential future threats from this highly contagious virus and the possibility of sudden large outbreaks involving a large number of SARS patients.

\section{REFERENCES}

1. World Health Organization. Acute respiratory syndrome China, Hong Kong Special Administrative Region of China, and Viet Nam. Wkly Epidemiol Rec 2003:78:73-74.

2. Tsang KW, Ho PL, Ooi GC, et al. A cluster of cases of severe acute respiratory syndrome in Hong Kong. $N$ Engl J Med 2003;348:19771985.

3. Lee N, Hui D, Wu A, et al. A major outbreak of severe acute respiratory syndrome in Hong Kong. N Engl J Med 2003;348:1986-1994.

4. Centers for Disease Control and Prevention. Severe acute respiratory syndrome: Singapore, 2003. MMWR 2003;52:405-411.

5. Poutanen SM, Low DE, Henry B, et al. Identification of severe acute respiratory syndrome in Canada. $N$ Engl J Med 2003;348:1995-2005.

6. Twu SJ, Chen TJ, Chen CJ, et al. Control measures for severe acute respiratory syndrome (SARS) in Taiwan. Emerg Infect Dis 2003;9:718720 .

7. World Health Organization. Cumulative Number of Reported Probable Cases of SARS From 1st November 2002 to 4th July 2003. Geneva: World Health Organization; 2003. Available at www. who.int/csr/sars/country2003 07 04/en/.

8. Centers for Disease Control and Prevention. Severe acute respiratory syndrome: Taiwan, 2003. MMWR 2003;52:461-466.

9. World Health Organization. Case Definitions for Surveillance of Severe Acute Respiratory Syndrome (SARS); 2003. Geneva: World Health Organization; 2003. Available at www.who.int/csr/sars/ casedefinition/en/. Accessed April 20, 2003.

10. Rainer TH, Cameron PA, Smit D, et al. Evaluation of WHO criteria for identifying patients with severe acute respiratory syndrome out of hospital: prospective observational study. Br Med I 2003;326:13541358. 
11. Pang X, Zhu Z, Xu F, et al. Evaluation of control measures implemented in the severe acute respiratory syndrome outbreak in Beijing, 2003. JAMA 2003;290:3215-3221.

12. Donnelly CA, Ghani AC, Leung GM, et al. Epidemiological determinants of spread of causal agent of severe acute respiratory syndrome in Hong Kong. Lancet 2003;361:1761-1766.

13. Riley S, Fraser C, Donnelly CA, et al. Transmission dynamics of the etiological agent of SARS in Hong Kong: impact of public health interventions. Science 2003;300:1961-1966.

14. World Health Organization. Hospital Infection Control Guidance for Severe Acute Respiratory Syndrome (SARS) (Revised 24 April 2003). Geneva: World Health Organization; 2003. Available at www. who.int/csr/sars/infectioncontrol/en/print.html.

15. Huang KY. The lessons of SARS. J Microbiol Immunol Infect 2003;36: 77-80.

16. World Health Organization. Summary of Probable SARS Cases With Onset of Illness From I November 2002 to 31 July 2003 (Revised 26 September 2003). Geneva: World Health Organization; 2003. Available at www. who.int/csr/sars/country/table2003_09_23/en/.

17. Seto WH, Tsang D, Yung RW, et al. Effectiveness of precautions against droplets and contact in prevention of nosocomial transmission of severe acute respiratory syndrome (SARS). Lancet 2003;361:15191520 .

\section{Medical News \\ Edited by Gina Pugliese, RN, MS; Martin S. Favero, PhD}

\section{Enhanced Identification of Postoperative Infections Among Inpatients and Outpatients}

Two recent studies address the enhanced identification of postoperative infections among inpatients and outpatients with review of charts for discharge diagnosis codes, antimicrobial use, or administration claims data.

In the first study, Yokoe et al. of Brigham and Women's Hospital, Boston, ${ }^{1}$ and researchers from 13 hospitals affiliated with the Centers for Disease Control and Prevention epicenters collaborated to study the ability of exposure to antimicrobial drugs and coded discharge diagnoses to identify surgical-site infections (SSIs) after three common procedures: coronary artery bypass graft $(\mathrm{CABG})$, cesarean delivery, and breast procedures. These measures were chosen because nearly all hospitals collect this information as part of routine patient care and many hospitals store this information.

This retrospective cohort study in 13 hospitals involved weighted, random samples of records from 8,739 CABG procedures, 7,399 cesarean deliveries, and 6,175 breast procedures. Routine surveillance was compared with detected inpatient antimicrobial exposure $\geqslant 9$ days for $\mathrm{CABG}, \geqslant 2$ days for cesarean deliveries, and $\geqslant 6$ days for breast procedures), discharge diagnoses, or both. Together, all methods identified SSI after $7.4 \%$ of CABG procedures, $5.0 \%$ of cesarean deliveries, and $2.0 \%$ of breast procedures. Antimicrobial exposure had the highest sensitivity, $88 \%$ to $91 \%$, compared with routine surveillance, $38 \%$ to $64 \%$. Diagnosis codes improved sensitivity of detection of antimicrobial exposure after cesarean deliveries. Record review confirmed SSI after $31 \%$ to $38 \%$ of procedures that met antimicrobial surveillance criteria. The authors con- cluded that sufficient antimicrobial exposure days, together with diagnosis codes for cesarean deliveries, identified more postoperative SSIs than did routine surveillance methods. In addition, this screening method was found to be efficient, readily standardized, and suitable for most hospitals.

In the second study, Miner et al. ${ }^{2}$ used administrative claims data to identify SSIs after breast surgery and cesarean section. Postoperative diagnosis codes, procedure codes, and pharmacy information were automatically scanned and used to identify claims suggestive of SSI ("indicators") among $426(22 \%)$ of 1,943 breast procedures and $474(10 \%)$ of 4,859 cesarean sections.

For 104 breast procedures with indicators explained in available medical records, SSIs were confirmed for $37 \%$, and some infection criteria were present for another $27 \%$. Among 204 cesarean sections, SSIs were confirmed for $40 \%$, and some criteria were met for $27 \%$. The extrapolated infection rates of $2.8 \%$ for breast procedures and $3.1 \%$ for cesarean sections were similar to those reported by the National Nosocomial Infections Surveillance System, but differed in representing predominantly outpatient infections. The authors concluded that claims data may complement other data sources for identification of SSIs following breast surgery and cesarean section.

FROM: 1. Yokoe DS, Noskin GA, Cunningham SM, et al. Enhanced identification of postoperative infections among inpatients. Emerg Infect Dis 2004;10:1924-1930. 2. Miner AL, Sands KE, Yokoe DS, et al. Enhanced identification of postoperative infections among outpatients. Emerg Infect Dis 2004;10:1931-1937. 